\title{
Perioperative Application of Diltiazem in Patients Undergoing On-Pump Coronary Artery Bypass Grafting
}

Xiaopeng Zhang ( $\nabla$ xzhang1@geisinger.edu )

Geisinger Medical Center

Yirui Hu

Geisinger Medical Center

Michael Friscia

Geisinger Medical Center

Xianren Wu

Geisinger Medical Center

Li Zhang

Geisinger Medical Center

Alfred Casale

Geisinger Medical Center

\section{Research Article}

Keywords: coronary artery bypass grafting, cardiopulmonary bypass, calcium channel blocker, arterial grafts, spasm

Posted Date: January 5th, 2022

DOI: https://doi.org/10.21203/rs.3.rs-1219497/v1

License: (c) (1) This work is licensed under a Creative Commons Attribution 4.0 International License. Read Full License 


\section{Abstract}

Diltiazem is frequently applied during the perioperative period of coronary artery bypass grafting with cardiopulmonary bypass (on-pump CABG) to prevent arterial graft spasm; however, the short- and longterm outcome effects are unclear. In this retrospective cohort study, from October 2008 to October 2018, adult patients who underwent isolated CABG with cardiopulmonary bypass (CPB) were identified. Patients in the perioperative diltiazem (DILT) and non-intervention (non-DILT) cohorts were matched based on propensity scores. Logistic regression was fitted to evaluate the impact on short-term outcomes. Long-term outcome was investigated by survival analyses. Total of 1004 patients were included. There were no statistically significant differences between the cohorts in perioperative outcomes, although at 24 hours postoperatively, patients in DILT were more likely to be on hemodynamic support from inotropic and/or vasoactive agents. The overall long-term survival was similar between the cohorts; however, in the subgroup of patients who received more than one arterial graft, perioperative diltiazem may be associated with higher long-term all-cause mortality. Based on these data, the perioperative and long-term outcome benefits of continuous iv infusion of diltiazem in patients who underwent on-pump CABG.

\section{Introduction}

The application of arterial grafts in coronary artery bypass grafting (CABG), most frequently left internal thoracic artery (LITA, previously internal mammary artery, LIMA) and/or radial arterial (RA) grafts, has apparent advantages over venous grafts ${ }^{1}$. Unlike venous grafts, which develop lumen stenosis over time due to subintimal fibrosis, arterial grafts have a longer period of lumen patency because of better size adaptability to native coronary vessels, more physiological flow from the elasticity of the vessel wall and regular lumen ${ }^{2}$. However, the significant arterial graft spasm of LITA and RA conduits may be responsible for cardiac ischemia due to graft failure ${ }^{3}$. In fact, the observed significant spasm of RA grafts by Carpentier et al., the very group that promoted this technique, resulted in a nearly two-decade delay in the regular clinical application of RA grafts ${ }^{4,5}$. Although not considered standard practice, expert opinion is accepted that perioperative application of selective coronary vasodilatory agents may help prevent arterial graft spasm, hence improving the outcomes.

Over the past three decades, numerous drugs have been investigated in RCTs for preventing graft spasm $^{6}$, among which certain calcium channel blockers (CCBs), e.g., diltiazem and nitroglycerin, were the top choices because of their well-known coronary selectivity and their proven track records of reliable symptomatic relief of unstable angina. Data from these RCTs, mostly conducted between the 1980s and early 2000s with small sample sizes on LITA grafts, suggested that, by direct flow check intraoperatively, both diltiazem and nitroglycerin increased arterial graft blood flow when applied locally and/or systemically, while when applied through continuous intravenous (iv) infusion perioperatively, diltiazem was superior to nitroglycerin for lower incidences of perioperative cardiac ischemia and arrythmia ${ }^{7-15}$. Currently, perioperative continuous iv infusion of diltiazem is frequently chosen as the intervention for 
preventing arterial graft spasms, especially for patients who receive RA grafts; however, its perioperative and long-term outcome benefits remain unconfirmed.

The purpose of this single-center propensity matched cohort study was to investigate the impacts of perioperative systemic application of diltiazem on perioperative and long-term outcomes in adult patients undergoing isolated $C A B G$ with cardiopulmonary bypass (CPB), e.g., on-pump CABG.

\section{Patients And Methods}

The protocol of this retrospective cohort study was approved by the institutional review board of Geisinger Medical Center. The approval number was 20180418. All methods were performed in accordance with relevant local, state, national, and institutional guidelines and regulations for human research. The exemption of informed consent was granted by the institutional review board since there was no risk of identity exposure.

Using designated search phrases (see Appendix 1), the Geisinger electronic health records and the Society for Thoracic Surgeons (STS) database were searched from October 2008 to October 2018 for adult patients undergoing isolated on-pump CABG. After deidentification, patient data were transferred to the principal investigator, $\mathrm{XZ}$, who determined the accessibility of each coinvestigator.

Two thousand nine hundred and sixteen consecutive adult patients undergoing elective or nonelective isolated on-pump CABG with at least one arterial graft were identified. Patients who underwent CABG combined with other open chamber procedures, aortic procedures and/or carotid endarterectomy were excluded. Depending on whether diltiazem was received perioperatively, the patients were assigned to two cohorts: the diltiazem cohort (DILT) and the control cohort (non-DILT). Propensity score matching (1:1) was performed based on the following patient characteristics: age, sex, year of surgery, STS morbidity and mortality score and the number of arterial grafts used. The primary outcomes were 30-day and longterm all-course mortalities; secondary outcomes included postoperative cardiac ischemia, new-onset atrial fibrillation (A-fib), stroke/transient ischemic attack (TIA), acute renal failure, multiple organ failure, cardiac arrest, length of mechanical ventilation, intensive care unit (ICU), hospital stay, and likelihood of home discharge. Subgroup analyses of the same outcomes were conducted for patients who received more than one arterial graft and those who had an intra-aortic balloon pump (IABP) inserted prior to CABG.

Perioperative management and diltiazem application

All routinely prescribed medications were continued until the day of surgery except oral anticoagulants, angiotensin-converting enzyme inhibitors, angiotensin receptor blockers and oral diabetic medications. iv infusion of nitroglycerin and/or heparin was discontinued after the patients were moved into the operating room. Patients were pre-medicated with Midazolam. Depending on the patient's ventricular function and the pathology of the coronary vessels, general anesthesia was induced with the combination of fentanyl with propofol, etomidate or midazolam, titrating to maintain hemodynamic 
stability. General anesthesia was maintained on isoflurane and rocuronium supplemented with fentanyl, midazolam and inotropic and/or vasoactive agents as needed. Standard protocols were followed for taking down the left and/or right LITA and dissecting peripheral arterial segments and saphenous venous segments. The grafts were preserved in Ringer's lactate solution containing verapamil $2.5 \mathrm{mg} / \mathrm{ml}$, nitroglycerin $5 \mathrm{mg} / \mathrm{ml}$ and heparin 1000 units $/ \mathrm{ml}$ until ready to be sutured onto the target native coronary vessels. During revascularization, the heart was protected by CPB with hypothermia and the perfusion of cardioplegia solution with a high potassium concentration. Continuous iv infusion of diltiazem at a dosage of $5 \mathrm{mg} / \mathrm{hr}$ was started after successfully weaning off CPB. For those cases that were converted from off-pump to on-pump CABG, diltiazem infusion was started upon sternal incision. The iv infusion was converted to oral diltiazem $120 \mathrm{mg}$ daily after patients were extubated and able to take medications orally. Oral diltiazem was typically discontinued after 30 days for LITA grafts and 6 months for radial artery grafts.

\section{Statistical Analysis}

Baseline characteristics are summarized as the mean \pm standard deviation (SD) for continuous variables and frequency for categorical variables. To estimate the treatment effect of DILT in an observational study, propensity score-based methods were used to account for covariate imbalance between DILT and non-DILT groups. One-to-one matching using nearest neighbors with a caliper of 0.20 based on propensity score was performed, including age, sex, Society of Thoracic Surgeons (STS) morbidity and mortality score, number of arterial grafts used, and surgical year (2008-2018) were included to estimate the propensity score. In the propensity score-matched cohorts, we further checked the balance of covariates using a covariate balance plot with a standardized mean difference (SMD) of $<0.20$. Unadjusted comparisons were performed for both categorical and continuous outcomes between the matched DILT and non-DILT groups. Pearson chi-square tests for independence were used to test whether the distribution of categorical outcomes differed between groups. Logistic regression models were used to evaluate the impact of diltiazem on adverse outcomes such as short-term mortality, new onset A-fib, cardiac ischemia, and stroke/TIA after adjusting for age, sex, STS morbidity and mortality score, and number of arterial grafts used. Incidence rate ratios (IRRs) with corresponding $95 \%$ confidence intervals (Cls) were reported for the treatment effect of DILT on adverse outcomes. Kaplan-Meier curves of time to mortality are presented for the propensity score matched cohort, stratified by the DILT and non-DILT groups. Statistical analyses were conducted in RStudio (Version 1.3.1093) using the Matchlt package ${ }^{16-}$ 18. P-values of less than 0.05 were considered statistically significant.

\section{Results}

Propensity Score matched cohorts

Two thousand nine hundred sixteen patients who underwent isolated on-pump CABG were included from October 2008 to October 2018. Among those, 617 were with DILT, and 2299 were with non-DILT cohorts. Patients in the DILT cohort were younger $(63.90 \pm 10.11$ vs. $65.66 \pm 9.94, p<0.001)$ and had a higher 
number of arterial grafts used $(1.33 \pm 0.84$ vs. $0.79 \pm 0.68, p<0.001)$. The DILT cohort had a significantly higher percentage of patients who received two or more arterial grafts $(6.3 \%$ vs. $1.6 \%, p<0.001)$.

Five hundred and two pairs of DILT and non-DILT patients were matched based on propensity score. The covariates were well balanced, with a standardized mean difference of $<0.10$ for covariates including age, sex, STS morbidity and mortality score (Appendix 2). Table 1 summarizes the patient characteristics before and after propensity score matching. 
Table 1

patient characteristics before and after propensity match

\section{Original Cohort}

PSM Cohort

\begin{tabular}{|c|c|c|c|c|c|c|}
\hline \multirow[b]{2}{*}{ General characteristics } & \multicolumn{3}{|c|}{ Original Cohort } & \multicolumn{3}{|l|}{ PSM Cohort } \\
\hline & $\begin{array}{l}\text { Non DILT } \\
(\mathrm{n}=2298)\end{array}$ & $\begin{array}{l}\text { DILT }(n= \\
617)\end{array}$ & $\begin{array}{l}\mathrm{p}- \\
\text { value }\end{array}$ & $\begin{array}{l}\text { Non DILT } \\
(n=502)\end{array}$ & $\operatorname{DILT}(\mathrm{n}=502)$ & $\begin{array}{l}\mathrm{p}- \\
\text { value }\end{array}$ \\
\hline Age (mean (SD)) & 65.65 (9.92) & $\begin{array}{l}63.90 \\
(10.11)\end{array}$ & $<0.001$ & $\begin{array}{l}64.84 \\
(9.75)\end{array}$ & $\begin{array}{l}64.53 \\
(10.26)\end{array}$ & 0.618 \\
\hline Male (\%) & $1766(76.8)$ & $\begin{array}{l}472 \\
(76.5)\end{array}$ & 0.9 & $394(78.5)$ & $380(75.7)$ & 0.33 \\
\hline STS score for mortality & $2.09(3.48)$ & $\begin{array}{l}1.93 \\
(2.99)\end{array}$ & 0.32 & $\begin{array}{l}1.99 \\
(3.91)\end{array}$ & $2.10(3.23)$ & 0.631 \\
\hline $\begin{array}{l}\text { STS score for } \\
\text { morbidity \& mortality }\end{array}$ & $\begin{array}{l}14.99 \\
(12.24)\end{array}$ & $\begin{array}{l}14.19 \\
(11.92)\end{array}$ & 0.15 & $\begin{array}{l}14.74 \\
(11.99)\end{array}$ & $\begin{array}{l}14.80 \\
(12.47)\end{array}$ & 0.933 \\
\hline \multicolumn{7}{|l|}{ Major comorbidities } \\
\hline Hypertension (\%) & $2061(89.7)$ & $\begin{array}{l}524 \\
(84.9)\end{array}$ & 0.001 & $442(88.0)$ & $426(84.9)$ & 0.17 \\
\hline COPD (\%) & $580(25.2)$ & $\begin{array}{l}223 \\
(36.1)\end{array}$ & $<0.001$ & $111(22.1)$ & $189(37.6)$ & $<0.001$ \\
\hline History of smoking (\%) & $250(10.9)$ & $59(9.6)$ & 0.39 & $52(10.4)$ & $45(9.0)$ & 0.52 \\
\hline $\begin{array}{l}\text { Peripheral vascular } \\
\text { disease (\%) }\end{array}$ & $344(15.0)$ & $\begin{array}{l}98 \\
(15.9)\end{array}$ & 0.62 & $65(12.9)$ & $81(16.1)$ & 0.18 \\
\hline Stroke/TIA (\%) & $512(22.3)$ & $\begin{array}{l}119 \\
(19.3)\end{array}$ & 0.12 & $98(19.5)$ & $104(20.7)$ & 0.69 \\
\hline \multicolumn{7}{|l|}{$\begin{array}{l}\text { Preoperative } \\
\text { medications }\end{array}$} \\
\hline ACE-I/ARB (\%) & $624(27.2)$ & $\begin{array}{l}207 \\
(33.5)\end{array}$ & 0.002 & $139(27.7)$ & $164(32.7)$ & 0.099 \\
\hline Aspirin (\%) & $2079(90.5)$ & $\begin{array}{l}554 \\
(89.8)\end{array}$ & 0.67 & $458(91.2)$ & $447(89.0)$ & 0.29 \\
\hline ß-blocker (\%) & $2105(91.6)$ & $\begin{array}{l}571 \\
(92.5)\end{array}$ & 0.5 & $472(94.0)$ & $462(92.0)$ & 0.27 \\
\hline $\begin{array}{l}\text { Calcium channel } \\
\text { blocker (\%) }\end{array}$ & $269(11.7)$ & $\begin{array}{l}79 \\
(12.8)\end{array}$ & 0.5 & 49 ( 9.8$)$ & $68(13.5)$ & 0.077 \\
\hline Nitrate (\%) & $222(9.7)$ & $47(7.6)$ & 0.14 & 49 ( 9.8$)$ & $40(8.0)$ & 0.37 \\
\hline \multicolumn{7}{|l|}{$\begin{array}{l}\text { Procedure related } \\
\text { characteristics }\end{array}$} \\
\hline $\begin{array}{l}\text { Number of arterial } \\
\text { graft (mean (SD)) }\end{array}$ & $0.79(0.68)$ & $\begin{array}{l}1.33 \\
(0.84)\end{array}$ & $<0.001$ & $\begin{array}{l}1.13 \\
(0.66)\end{array}$ & $1.10(0.67)$ & 0.57 \\
\hline
\end{tabular}




\begin{tabular}{|c|c|c|c|c|c|c|}
\hline \multirow[b]{2}{*}{ IABP (\%) } & \multicolumn{3}{|c|}{ Original Cohort } & \multicolumn{3}{|c|}{ PSM Cohort } \\
\hline & $535(15.9)$ & $89(9.4)$ & $<0.001$ & 75 (14.9) & $54(10.8)$ & 0.059 \\
\hline $\begin{array}{l}\text { Prolonged mechanical } \\
\text { ventilation (\%) }\end{array}$ & $204(8.9)$ & $44(7.1)$ & 0.19 & $32(6.4)$ & $40(8.0)$ & 0.39 \\
\hline $\begin{array}{l}\text { CBP time } \\
\text { (median(IQR)) }\end{array}$ & $83(65-107)$ & $\begin{array}{l}92(75- \\
111)\end{array}$ & $<0.001$ & $\begin{array}{l}83(65- \\
106)\end{array}$ & $91(74-111)$ & $<0.001$ \\
\hline
\end{tabular}

Perioperative outcomes

As shown in Table 2, based on logistic regression models, there were no significant differences in the prevalence rates of 30-day all-cause mortality (IRR: $2.33,95 \% \mathrm{Cl}: 0.91$ to $5.96, p=0.07$ ), postoperative cardiac ischemia (IRR: $1.10,95 \% \mathrm{Cl}: 0.60$ to $2.02, \mathrm{p}=0.75$ ), new onset A-fib (IRR: $1.06,95 \%$ : 0.78 to 1.43 , $\mathrm{p}=0.73$ ), or stroke/TIA (IRR: $0.76,95 \% \mathrm{Cl}: 0.17$ to $3.38, p=0.71$ ) between cohorts after adjusting for age, sex, STS morbidity and mortality score, and number of arterial grafts used. In addition, we estimated the number needed to harm $(\mathrm{NNH})$, which corresponds to the number of individuals who must be treated so that one of them presents an adverse reaction accountable to the treatment: for A-fib, $\mathrm{NNH}=126$; for 30day all-cause mortality, $\mathrm{NNH}=63$; for postoperative cardiac ischemia, $\mathrm{NNH}=167$. The prevalence rates of postoperative acute renal failure, multiple organ failure and cardiac arrest were comparable between cohorts.

Table 2

Perioperative outcomes of diltiazem in patients who received isolated on-pump CABG.

\begin{tabular}{|llll|}
\hline & non-DILT (n=502) & DILT (n=502) & p-value \\
\hline All-cause mortality (\%) & $59(11.8)$ & $67(13.3)$ & 0.505 \\
\hline 30-day mortality (\%) & $7(1.4)$ & $15(3.0)$ & 0.131 \\
\hline Atrial fibrillation (\%) & $81(16.1)$ & $85(16.9)$ & 0.799 \\
Cardiac ischemia (\%) & $20(4.0)$ & $23(4.6)$ & 0.755 \\
\hline Stroke/TIA (\%) & $4(0.8)$ & $3(0.6)$ & - \\
\hline Hours of ICU (mean (SD)) & $47.42(65.78)$ & $51.76(86.83)$ & 0.372 \\
\hline Inpatient days (mean (SD)) & $8.18(4.04)$ & $8.45(5.41)$ & 0.36 \\
\hline p-value: based on Pearson Chi-Squared tests. & & \\
\hline
\end{tabular}

Perioperative inotropic and/or vasoactive agent dependency

The differences between the start and end times were estimated for the inotropic and/or vasoactive agents and converted into binary variables using 24 hours as the cut-off timepoint. Among the 502 pairs, 
136 observations had over 24-hour inotropic and/or vasoactive agent usage in DILT and 59 observations in non-DILT. The chi-squared test suggested that patients on perioperative diltiazem were more likely to be on prolonged inotropic and/or vasoactive agents 24 hours postoperatively $(p<0.0001)$ (Fig. 1).

Lengths of cares and final discharge location

There was no significant difference in the length of mechanical ventilation, length of intensive care unit stay or total days of inpatient care. Among the 502 pairs, 427 observations in DILT and 385 in non-DILT were discharged to home. The chi-squared test revealed that patients in the DILT cohort were more likely to be discharged home $(p=0.001)$ (Fig. 2).

Survival analyses

Among the 502 matched pairs, 67 (13.3\%) died in DILT and 59 (11.8\%) in non-DILT over the postoperative follow-up period. Kaplan-Meier curves stratified by diltiazem over 60 months of follow-up showed that the curves crossed several times during the follow-up period, which indicated that there was no significant difference in long-term all-cause mortality between DILT and non-DILT (Fig. 3).

\section{Subgroups analyses}

Table 3 summarizes the perioperative outcomes of the subgroup of patients who received two or more arterial grafts. There was no significant difference in the incidences of 30-day all-cause mortality, total death over time, new onset A-fib, cardiac ischemia, stroke/TIA, length of ICU stay, or total inpatient days between DILT and non-DILT. The log-rank test indicated that perioperative diltiazem may be associated with less long-term survival ( $p=0.02)$ (Fig. 4). 
Table 3

Perioperative outcomes diltiazem in subgroup of patients who received two or more arterial grafts

\begin{tabular}{|llll|}
\hline & non-DILT (n=114) & DILT (n=124) & p-value \\
\hline All-cause mortality (\%) & $8(7.0)$ & $17(13.7)$ & 0.141 \\
\hline 30-day all-cause mortality (\%) & $1(0.9)$ & $2(1.6)$ & - \\
\hline Atrial fibrillation (\%) & $16(14.0)$ & $15(12.1)$ & 0.802 \\
\hline Cardiac ischemia (\%) & $4(3.5)$ & $3(2.4)$ & 0.91 \\
\hline Stroke/TIA (\%) & $0(0.0)$ & $1(0.8)$ & - \\
\hline Hours of ICU (mean (SD)) & $38.26(43.11)$ & $43.30(56.70)$ & 0.444 \\
\hline Inpatient days (mean (SD)) & $7.77(3.43)$ & $7.61(4.35)$ & 0.756 \\
\hline p-value: based on Pearson Chi-Squared tests. & & \\
\hline
\end{tabular}

Table 4 summarizes the perioperative outcomes of diltiazem in the subgroup of patients who had preoperatively inserted IABP. There were no significant differences in the incidences of 30-day all-cause mortality, total death over time, new onset A-fib, cardiac ischemia, stroke/TIA, length of ICU stay or total inpatient days between DILT and non-DILT. The log-rank test indicated that perioperative diltiazem had no significant effect on long-term survival (Fig. 5).

Table 4

Perioperative outcomes of diltiazem in the subgroup of patients who had preoperatively inserted IABP

\begin{tabular}{|llll|}
\hline & non-DILT (n=53) & DILT (n=53) & p-value \\
\hline All-cause mortality (\%) & $11(20.8)$ & $10(18.9)$ & - \\
\hline 30-day all-cause mortality (\%) & $1(1.9)$ & $4(7.5)$ & 0.36 \\
\hline Atrial fibrillation (\%) & $6(11.3)$ & $14(26.4)$ & 0.082 \\
\hline Cardiac ischemia (\%) & $4(7.5)$ & $2(3.8)$ & 0.674 \\
\hline Stroke/TIA (\%) & $2(3.8)$ & $0(0)$ & - \\
\hline Hours of ICU (mean (SD)) & $64.88(61.30)$ & $97.63(105.58)$ & 0.054 \\
\hline Inpatient days (mean (SD)) & $8.83(5.33)$ & $10.85(6.39)$ & 0.082 \\
\hline p-value: based on Pearson Chi-Squared tests. & & \\
\hline
\end{tabular}




\section{Discussion}

Arterial graft spasm may cause critical cardiac ischemia during and after CABG. The reported incidence of arterial graft spasm with compromised cardiac pump function and hemodynamic stability was between $0.5 \%$ and $1.3 \% 3,19,20$. Currently, the commonly accepted perioperative intervention to prevent arterial graft spasm is to locally and/or systemically apply vasodilators with coronary selectivity. Diltiazem and nitroglycerin became frequently chosen agents based on the results of RCTs conducted mostly in patients who received LITA grafts, with a very limited number of studies targeting arterial grafts of other sources, including RA grafts. However, the usage of RA grafts is usually the main reason for perioperative antispasmodic intervention because of the known high tendency of spasm of RA grafts. There were limited, nonrandomized control data of intraoperative direct flow checks indicating that continuous iv infusion of diltiazem and nitroglycerine increased blood flow in the LITA and RA grafts. However, there has been no definitive anatomical evidence supporting that perioperative continuous iv application of diltiazem had a direct vasodilatory effect on coronary grafts after the sternum was closed. Diltiazem became the preferred antispasmodic agent because, compared to nitroglycerine, it had more favorable perioperative outcome profiles, including lower incidences of new onset A-fib and cardiac ischemia $7,9,21,22$. Noticeably, data from these RCTs also suggest that patients on diltiazem may need prolonged hemodynamic support from inotropic and/or vasoactive support, indicating that the usage of diltiazem may have potential negative perioperative and long-term outcome impacts ${ }^{22}$.

Due to the limitation of the small sample sizes, there were no available data on short- and long-term mortalities reported from these RCTs. In fact, despite decades of perioperative diltiazem application for preventing graft spasm in CABG, there are scarcely available observational data of long-term outcomes after hospital discharge. In our institute, diltiazem has been routinely applied in patients who underwent on-pump CABG, especially those who received RA grafts. As part of the clinical quality improvement initiative, we conducted this retrospective study to validate the practice and explore the perioperative and long-term outcome effects of perioperative diltiazem in our patient population. To summarize the findings of this propensity matched cohort study, there were no significant differences in perioperative outcomes if diltiazem was applied. There was no significant difference in 30-day and long-term all-cause mortality. Patients who received preventive perioperative diltiazem had a significantly higher requirement for hemodynamic support from inotropic and/or vasoactive agents 24 hours postoperatively. In addition, data from the subgroup analyses revealed that diltiazem may be associated with reduced long-term survival in patients who received LITA with additional arterial grafts, which were mostly RA grafts. Based on these findings, we could not confirm that perioperative diltiazem had significant perioperative and long-term outcome benefits in our studied patient population.

Although due to the limitation of a single-center retrospective study, it is premature to conclude that diltiazem has no or negative benefits in patients who underwent on-pump CABG, we think the data from this study do have important clinical implications. 
The data of our study validated those from previously published studies showing that the vasodilatory and negative inotropic effects of diltiazem had significant perioperative hemodynamic consequences, which may negatively affect the perioperative outcomes due to reduced coronary perfusion pressures as well as perfusion pressure for other end organs. Studies have shown that the length and dosage of the application of perioperative inotropic and/or vasoactive agents were predictive of negative postoperative outcomes, including mortalities, in patients who underwent cardiac surgeries ${ }^{23}$. In our study, the calculated NNHs for several perioperative outcomes indicated that the potential side effects of continuous iv infusion of diltiazem may have to be taken into consideration for starting the intervention. We suspect that the perioperative hemodynamic instability associated with diltiazem may cause unfavorable effects on perioperative and long-term outcomes. One speculation for the negative long-term outcome in the subgroup of patients who received two or more arterial grafts is that the decreased coronary pressure from the systemic application of diltiazem might negate the antispasmodic effect. It may be reasonable to consider the combination of a preventive perioperative intra-aortic balloon pump (IABP) with perioperative diltiazem, rather than diltiazem alone, for better protection of cardiac ischemia caused by arterial graft spasm, although a well-designed randomized clinical trial would be necessary to test this hypothesis.

As mentioned above, the preventive application of diltiazem is based on the data from RCTs focused on LITA grafts; however, in practice, patients receiving RA grafts are more likely to be treated with perioperative diltiazem, assuming that the effect of diltiazem on all types of arterial grafts is the same. The dosage of diltiazem, $0.05 \sim 0.1 \mathrm{mg} / \mathrm{kg}$, is also based on the same studies. This could be an important confounding issue because studies have reported that different types of arterial grafts may, in fact, have different responses to diltiazem and other vasodilators. The majority of past studies have shown that LITA was more responsive to local and/or systemic applications of diltiazem with more graft dilation and blood flow $7,9,21,24,25$, and diltiazem had more perioperative outcome advantages than nitroglycerin in patients who received LITA grafts as the only arterial graft ${ }^{14,26-31}$. When RA or other arterial grafts are involved, however, diltiazem may not be the best choice. An earlier RCT by Guadino et al. suggested that long-term postoperative CCB was not associated with better RA graft patency and may not provide outcome benefits for patients who had RA grafts ${ }^{29}, 32$, although their recent study with questionable methodology argued against their previous conclusion: the post hoc analysis of data pooled from six RCTs comparing RA and SVG showed that chronic postoperative CCB therapy may improve RA graft patency and reduce the incidence of major cardiovascular events ${ }^{33}$. Numbers of in vitro and in vivo studies of RA sections or conduits indicated that nitroglycerin was more effective than diltiazem in preventing the contraction of RA grafts ${ }^{30,34}$; compared to other CCBs, diltiazem was less effective in preventing graft contraction caused by endothelin- 1 and norepinephrine, which affected RA more than LITA $^{28}$. Chanda et al. proposed that the combination of nitroglycerin and CCB may be more effective than a single agent for preventing RA graft spasm ${ }^{31}$. In addition, data from recent studies investigating various categories of vasodilators support that the antispasmodic effects may depend on the types of vessels and mechanisms of graft spasm ${ }^{35-39}$. Taken together, evidence suggests that instead of applying 
diltiazem to every patient, tailoring the antispasmodic interventions to the types and conditions of arterial grafts, as well the clinical condition of each individual patient, may be more effective. To better understand the effects of diltiazem on RA grafts, we think a large-scale, multicenter retrospective study and a well-designed, properly powered RCT investigating the preventive effects and outcome impacts of diltiazem may be warranted.

As a part of the quality improvement and cost-reducing study, we did compare the lengths of ICU admission and hospitalization as well as the likely discharge locations of patients who had or did not have perioperative diltiazem. Data suggested that the lengths of care were similar between cohorts; the patients who received diltiazem were more likely to be discharged to home instead of cardiac rehabilitation facilities, suggesting diltiazem cohort may have improved social and economic outcomes, however, we cannot draw a conclusion unless it can be proved by a well-conducted clinical trial.

Our study does have important limitations. First, like other retrospective clinical studies, selection bias cannot be eliminated. Considering the limited sample volume, we were not able to include every covariate in the PSM; therefore, it is possible that certain patient baseline and perioperative characteristics were unequal between matched cohorts, and some of the unmatched covariates could be potential confounding factors for the studied outcomes. We noticed that the DILT cohort had a significantly higher percentage of patients who had a history of COPD, longer cardiopulmonary bypass time, and higher tendency to receive two or more arterial grafts. Although unconfirmed, it was also possible that there was a tendency of more frequently applying perioperative iv infusion of diltiazem in patients whose graft condition was not reassuring judged by the visualization and the Doppler flow check. Second, while previous RCTs enrolled patients undergoing elective CABG only, the current study included patients undergoing both elective and urgent/emergent procedures. Patients who received RA or other arterial grafts in addition to LITA grafts and those who required insertion of IABP prior to planned CABG were included in our study because the severity of the coronary pathologies rendered these patients more vulnerable to graft spasm, and diltiazem therapy could be potentially more beneficial; however, those patients were mostly excluded from early RCTs. This could be an explanation why the favorable perioperative outcome effects observed were not reproduced. Thirdly, the study may be underpowered. Based on the observed data of this study, the post hoc estimated sample size for detecting differences in perioperative all-cause mortality was 1100 in each cohort; however, after PSM, the number of patients enrolled in each cohort was 502; therefore, the study may be underpowered for the investigation of certain perioperative outcomes. Finally, in the current study, the chosen starting rate of infusion for diltiazem was $5 \mathrm{mg} / \mathrm{hr}$, which fell within the range of $0.05 \sim 0.1 \mathrm{mg} / \mathrm{kg}$, the dosage used in earlier RCTs. However, the variation of the serum diltiazem concentrations due to the difference of body weights as well as that in the management of the infusion by ICU staff may affect the outcomes.

In conclusion, data from the current retrospective propensity matched cohort study did not validate the short- and long-term outcome benefits of perioperative diltiazem revealed by previous clinical studies. The preventive application of arterial graft spasm may have to be individualized, tailored to the types and conditions of the grafts as well as the patient's clinical conditions. We propose that a large-scale

Page $12 / 20$ 
multicenter retrospective outcome study and further clinical trials of diltiazem and/or other coronary selective vasodilators on RA grafts, in combination with treatment maintaining adequate coronary perfusion, may be warranted.

\section{Declarations}

Acknowledgement: We thank Matthew C. Gass, M.S for assisting data extraction and curation.

Funding: This work is supported by the Geisinger internal research fund.

Competing interest: The authors have no competing interests to report.

\section{References}

1. Gaudino, M. et al. Radial-Artery or Saphenous-Vein Grafts in Coronary-Artery Bypass Surgery. New England Journal of Medicine 378, 2069-2077 (2018).

2. Carpentier, A., Guermonprez, J. L., Deloche, A., Frechette, C. \& DuBost, C. The aorta-to-coronary radial artery bypass graft. A technique avoiding pathological changes in grafts. Ann. Thorac. Surg. 16, 111-121 (1973).

3. Harskamp, R. E. et al. Postoperative internal thoracic artery spasm after coronary artery bypass grafting. Ann. Thorac. Surg. 85, 647-649 (2008).

4. Geha, A. S., Krone, R. J., McCormick, J. R. \& Baue, A. E. Selection of coronary bypass. Anatomic, physiological, and angiographic considerations of vein and mammary artery grafts. Journal of Thoracic \& Cardiovascular Surgery 70, 414-431 (1975).

5. Acar, C. et al. Revival of the radial artery for coronary artery bypass grafting. Ann. Thorac. Surg. 54, 652-659 (1992).

6. He, G. W. \& Taggart, D. P. Spasm in Arterial Grafts in Coronary Artery Bypass Grafting Surgery. Ann. Thorac. Surg. 101, 1222-1229 (2016).

7. Erdem, 0. et al. Effects of intraoperative diltiazem infusion on flow changes in arterial and venous grafts in coronary artery bypass graft surgery. Revista Brasileira de Cirurgia Cardiovascular: Orgao Oficial da Sociedade Brasileira de Cirurgia Cardiovascular 30, 459-465 (2015).

8. Zabeeda, D. et al. Comparison of systemic vasodilators: effects on flow in internal mammary and radial arteries. Ann. Thorac. Surg. 71, 138-141 (2001).

9. Tabel, Y. et al. Diltiazem provides higher internal mammary artery flow than nitroglycerin during coronary artery bypass grafting surgery. European Journal of Cardio-Thoracic Surgery 25, 553-559 (2004).

10. Dobrilovic, N., Vadlamani, L., Buchert, B. \& Wright, C. B. Diltiazem prophylaxis reduces incidence of atrial fibrillation after coronary artery bypass grafting. J. Cardiovasc. Surg. 46, 457-461 (2005). 
11. Hannes, W. et al. Effect of peri-operative diltiazem on myocardial ischaemia and function in patients receiving mammary artery grafts. Eur. Heart J. 16, 87-93 (1995).

12. Pizzetti, G. et al. Beneficial effects of diltiazem during myocardial reperfusion: a randomized trial in acute myocardial infarction. Italian Heart Journal: Official Journal of the Italian Federation of Cardiology 2, 757-765 (2001).

13. Malhotra, R. et al. Cardioprotective effects of diltiazem infusion in the perioperative period. European Journal of Cardio-Thoracic Surgery 12, 420-427 (1997).

14. Seitelberger, R. et al. Effects of diltiazem on perioperative ischemia, arrhythmias, and myocardial function in patients undergoing elective coronary bypass grafting. J. Thorac. Cardiovasc. Surg. 107, 811-821 (1994).

15. Chanda, J. \& Canver, C. C. Reversal of preexisting vasospasm in coronary artery conduits. Ann. Thorac. Surg. 72, 476-480 (2001).

16. https://www.r-project.org/.

17. https://rstudio.com/products/team/.

18. Ho, D., Imai, K., King, G. \& Stuart, E. A. Matchlt: Nonparametric Preprocessing for Parametric Causal Inference. Journal of Statistical Software 42, 1-28 (2011).

19. Buxton, A. E., Goldberg, S., Harken, A., Hirshfield, J.,Jr \& Kastor, J. A. Coronary-artery spasm immediately after myocardial revascularization: recognition and management. N. Engl. J. Med. 304, 1249-1253 (1981).

20. Lorusso, R. et al. Refractory spasm of coronary arteries and grafted conduits after isolated coronary artery bypass surgery. Ann. Thorac. Surg. 93, 545-551 (2012).

21. Battaloglu, B. et al. Effects of pretreatment with different topical vasodilators on blood flow in the internal mammary artery: a prospective randomized study. Heart Surg. Forum 10, 136 (2007).

22. Hu, Y. et al. Perioperative diltiazem or nitroglycerin in on-pump coronary artery bypass: A systematic review and network meta-analysis. PLoS ONE [Electronic Resource] 13, e0203315 (2018).

23. Koponen, T. et al. Vasoactive-inotropic score and the prediction of morbidity and mortality after cardiac surgery. British journal of anaesthesia: BJA 122, 428-436 (2019).

24. Malhotra, R. et al. Cardioprotective effects of diltiazem infusion in the perioperative period. European Journal of Cardio-Thoracic Surgery 12, 420-427 (1997).

25. Hannes, W. et al. Effect of peri-operative diltiazem on myocardial ischaemia and function in patients receiving mammary artery grafts. Eur. Heart J. 16, 87-93 (1995).

26. Zvara, D. A. et al. Prophylactic nitroglycerin did not reduce myocardial ischemia during accelerated recovery management of coronary artery bypass graft surgery patients. Journal of Cardiothoracic \& Vascular Anesthesia 14, 571-575 (2000).

27. Shapira, O. M. et al. Nitroglycerin is superior to diltiazem as a coronary bypass conduit vasodilator. Journal of Thoracic \& Cardiovascular Surgery 117, 906-911 (1999). 
28. Chen, X. et al. [Perioperative changes of plasma ET-1 in patients undergoing coronary artery bypass grafting and the effect of nitroglycerin]. Chung-Hua Wai Ko Tsa Chih [Chinese Journal of Surgery] 38, 669-671 (2000).

29. Gaudino, M. et al. Is postoperative calcium channel blocker therapy needed in patients with radial artery grafts?. Journal of Thoracic \& Cardiovascular Surgery 129, 532-535 (2005).

30. Shapira, O. M. et al. Nitroglycerin is preferable to diltiazem for prevention of coronary bypass conduit spasm. Ann. Thorac. Surg. 70, 883-888 (2000).

31. Chanda, J., Brichkov, I. \& Canver, C. C. Prevention of radial artery graft vasospasm after coronary bypass. Ann. Thorac. Surg. 70, 2070-2074 (2000).

32. Gaudino, M., Glieca, F., Luciani, N., Alessandrini, F. \& Possati, G. Clinical and angiographic effects of chronic calcium channel blocker therapy continued beyond first postoperative year in patients with radial artery grafts: results of a prospective randomized investigation. Circulation 104, 64-67 (2001).

33. Gaudino, M. et al. Effect of Calcium-Channel Blocker Therapy on Radial Artery Grafts After Coronary Bypass Surgery. J Am Coll Cardiol 73, 2299-2306 (2019).

34. Fukuda, S. et al. Comparison of direct effects of clinically available vasodilators; nitroglycerin, nifedipine, cilnidipine and diltiazem, on human skeletonized internal mammary harvested with ultrasonic scalpel. Heart \& Vessels 31, 1681-1684 (2016).

35. He, G. W. \& Yang, C. Q. Inhibition of vasoconstriction by potassium channel opener aprikalim in human conduit arteries used as bypass grafts. Br. J. Clin. Pharmacol. 44, 353-359 (1997).

36. Liu, J. J., Doolan, L. A., Xie, B., Chen, J. R. \& Buxton, B. F. Direct vasodilator effect of milrinone, an inotropic drug, on arterial coronary bypass grafts. FANZCA. Journal of Thoracic \& Cardiovascular Surgery 113, 108-113 (1997).

37. Ertuna, E. et al. Effects of levosimendan on isolated human internal mammary artery and saphenous vein: concurrent use with conventional vasodilators. Fundam. Clin. Pharmacol. 30, 226-234 (2016).

38. Guzeloglu, M., Ertuna, E., Arun, M. Z. \& Reel, B. Effects of carvedilol on vascular reactivity in human left internal mammary artery. Eur Rev Med Pharmacol Sci 21, 4983-4988 (2017).

39. Bai, X., Liu, X., Yang, Q., Tang, X. \& He, G. The interaction between human urotensin II and vasodilator agents in human internal mammary artery with possible clinical implications. Ann Thorac Surg 92 , 610-616 (2011).

\section{Figures}




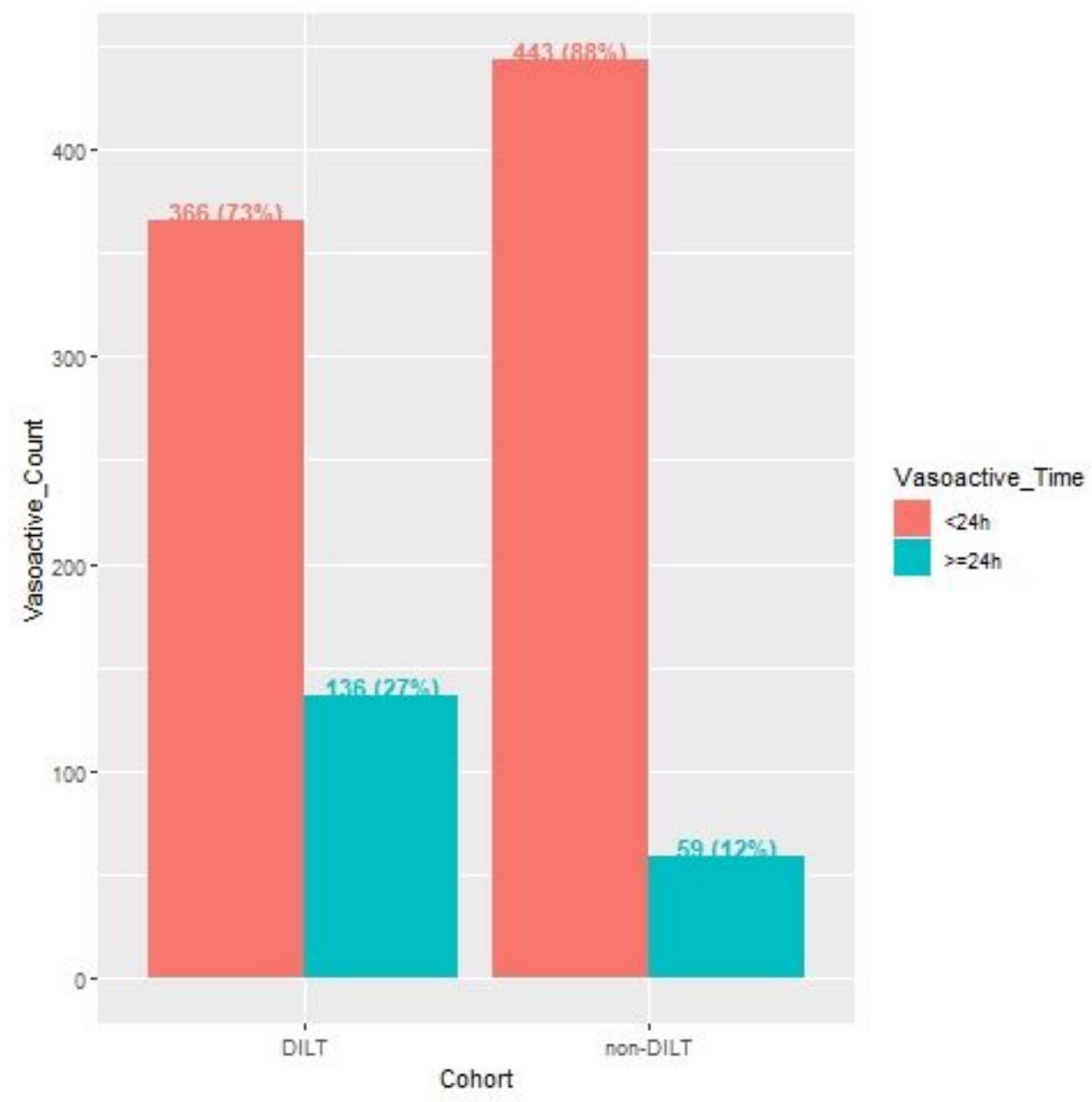

\section{Figure 1}

Bar chart of counts of patients on inotropic and/or vasoactive agents 24 hours postoperatively. Diltiazem was associated with longer inotropic/vasoactive support $(p<0.0001)$. 


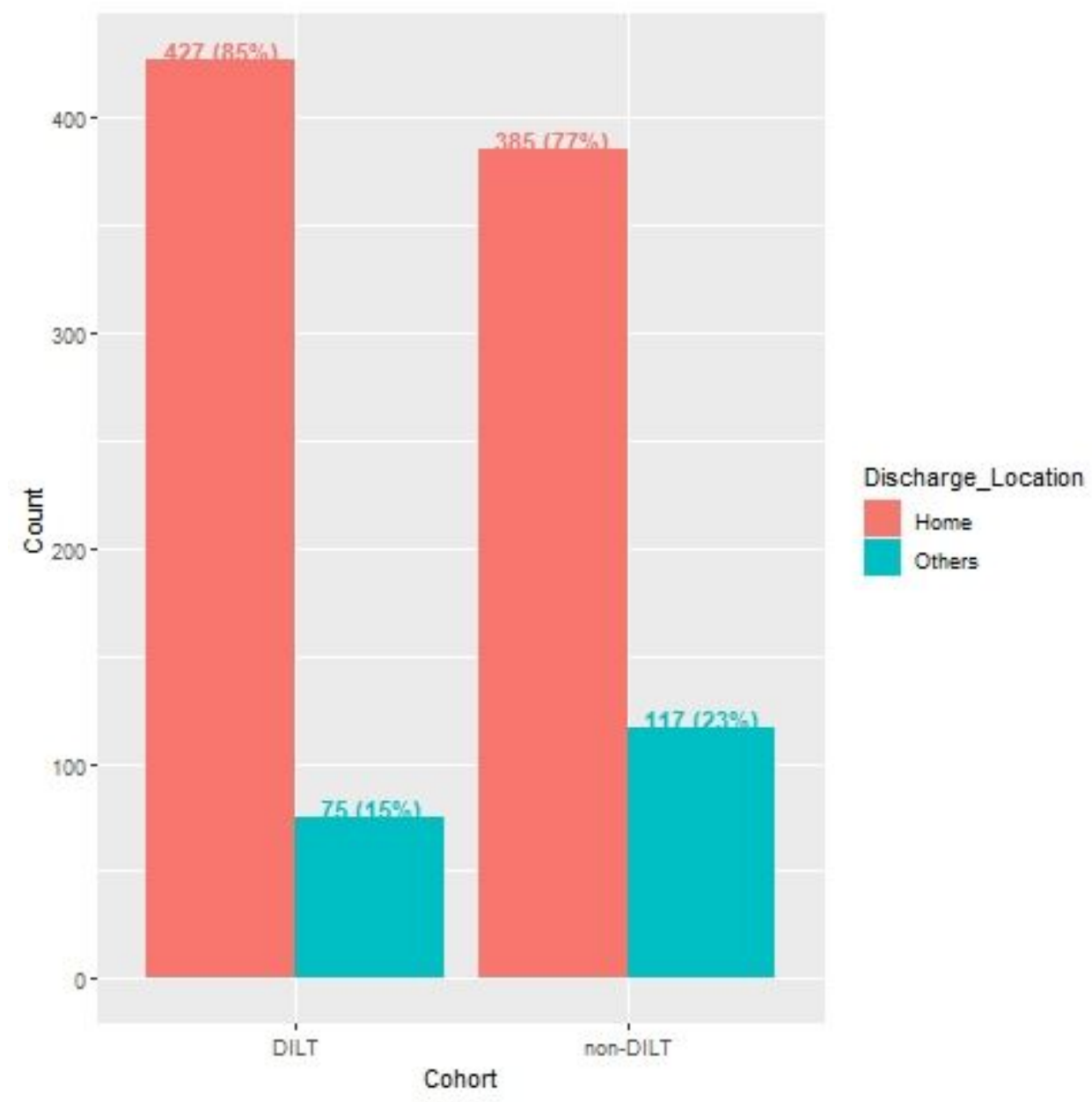

Figure 2

Bar chat of discharge locations. Diltiazem was associated with a higher likelihood of home discharge $(p=0.001)$. 


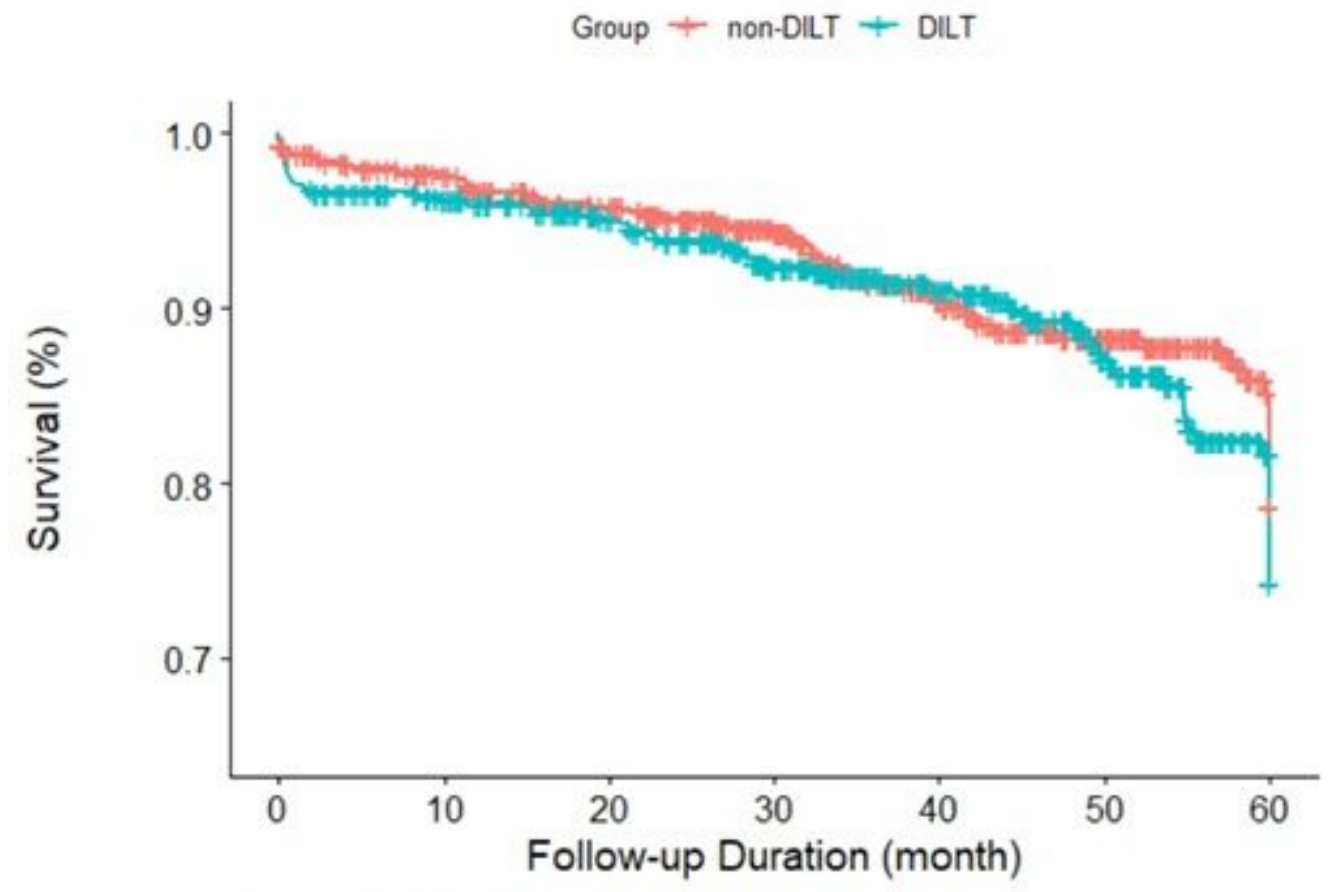

\begin{tabular}{|c|c|c|c|c|c|c|c|}
\hline \multicolumn{8}{|c|}{ Number at risk } \\
\hline 윽 non-DILT & 502 & 459 & 424 & 350 & 270 & 206 & 118 \\
\hline DILT. & 502 & 455 & 404 & 347 & 271 & 191 & 100 \\
\hline & 0 & 10 & 20 & 30 & 40 & 50 & 60 \\
\hline
\end{tabular}

Figure 3

Kaplan-Meier survival curve for overall survival of patients in the DILT and non-DILT cohorts.

Subgroups analyses 


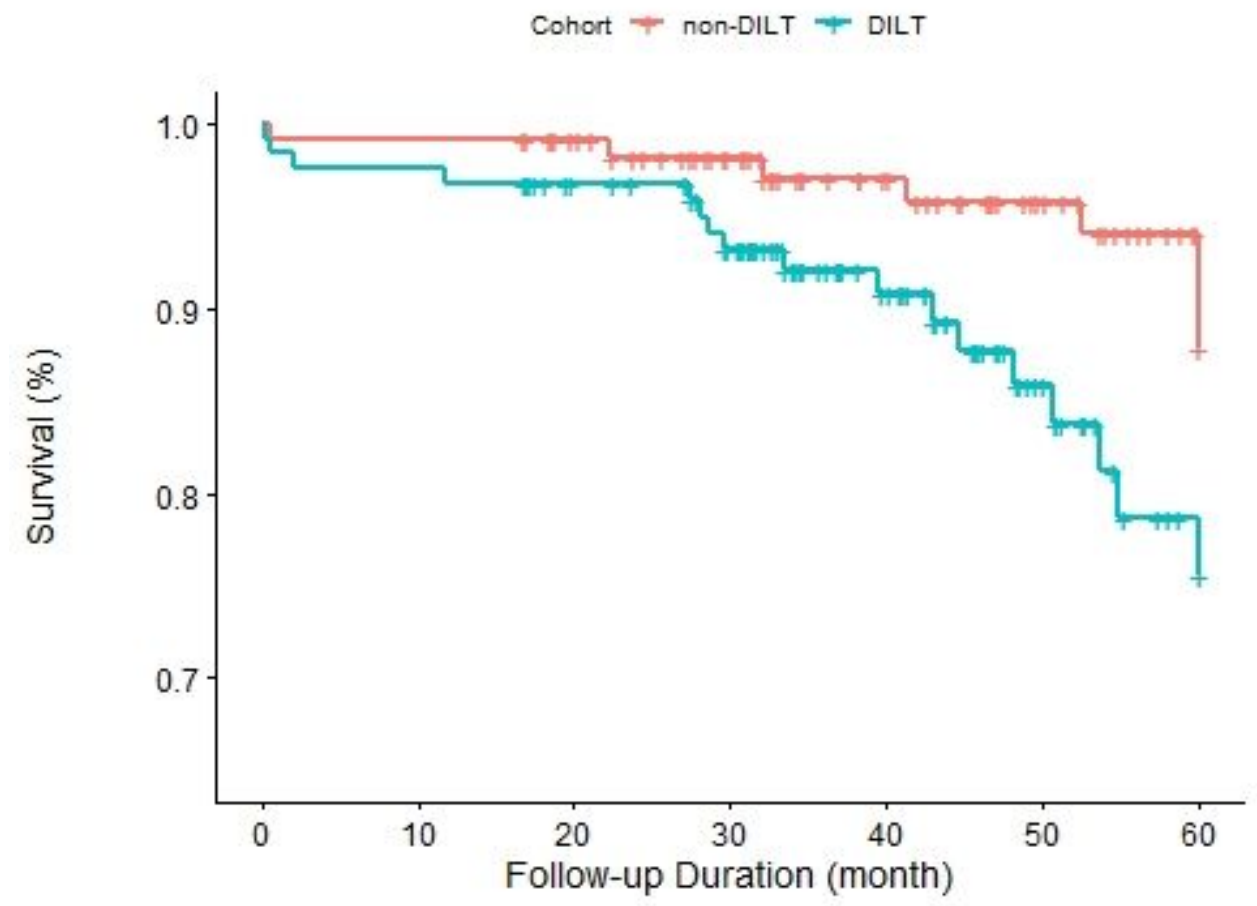

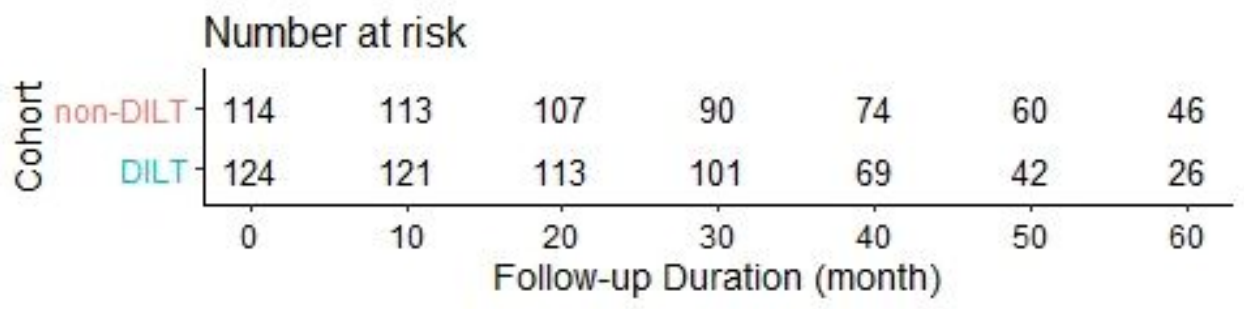

\section{Figure 4}

Kaplan-Meier survival curve for the subgroup of patients who received two or more arterial grafts. DILT had significantly lower long-term survival $(p=0.02)$. 


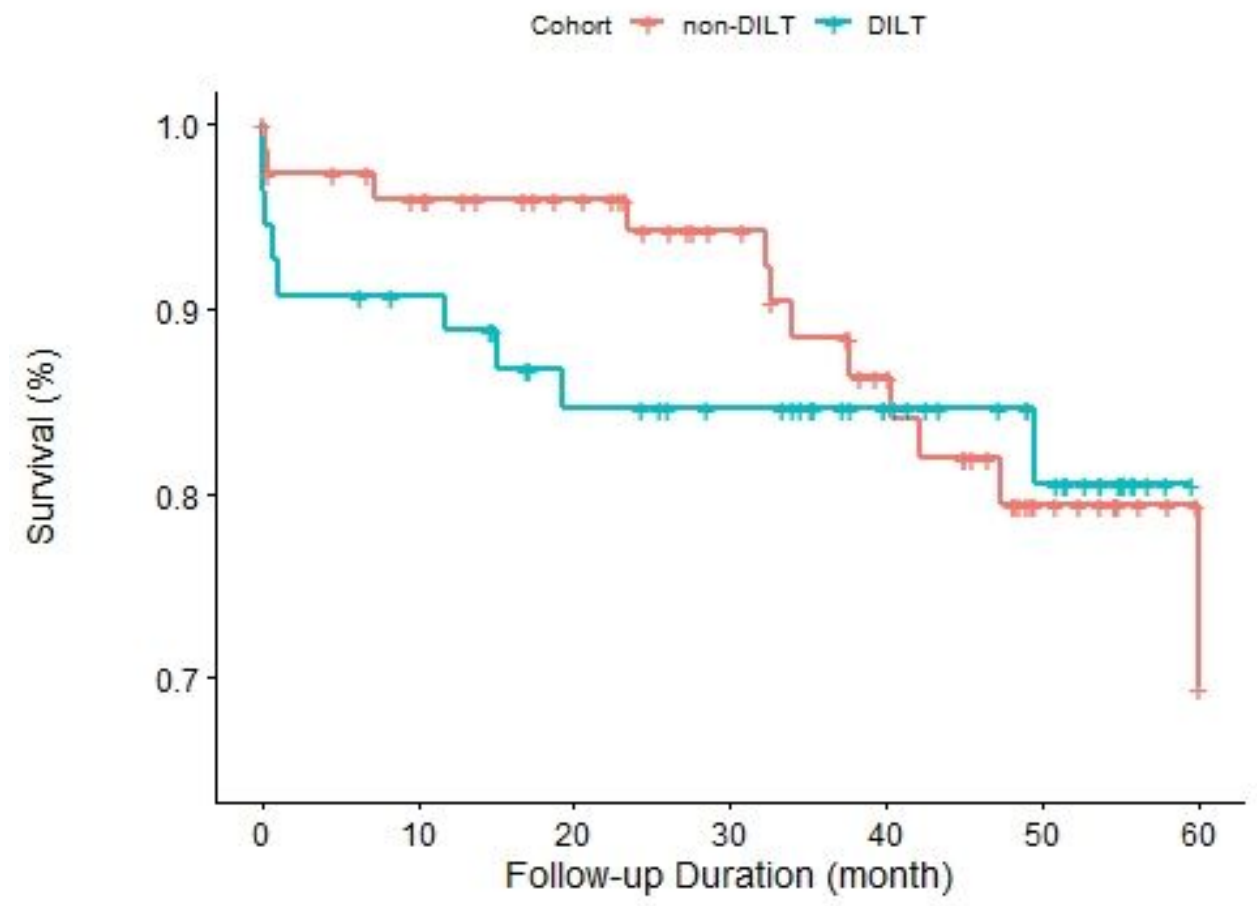

\begin{tabular}{|c|c|c|c|c|c|c|c|}
\hline \multicolumn{8}{|c|}{ Number at risk } \\
\hline \multirow{3}{*}{ 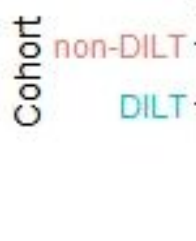 } & 75 & 67 & 60 & 50 & 40 & 25 & 16 \\
\hline & 54 & 47 & 40 & 36 & 27 & 19 & 4 \\
\hline & 0 & 10 & 20 & 30 & 40 & 50 & 60 \\
\hline
\end{tabular}

Figure 5

Kaplan-Meier survival curve for the subgroup of patients with IABP. There was no significant difference between cohorts.

\section{Supplementary Files}

This is a list of supplementary files associated with this preprint. Click to download.

- Appendix1.docx

- Appendix2.docx 